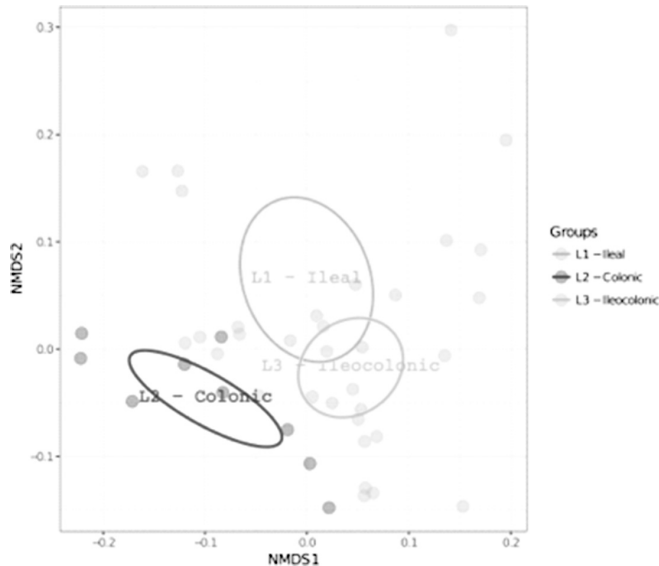

Abstract OWE-010 Figure 4 NMDS weighted UniFrac (bacteriabiopsies - disease distribution) $R^{2}=0.17 p=0.001$

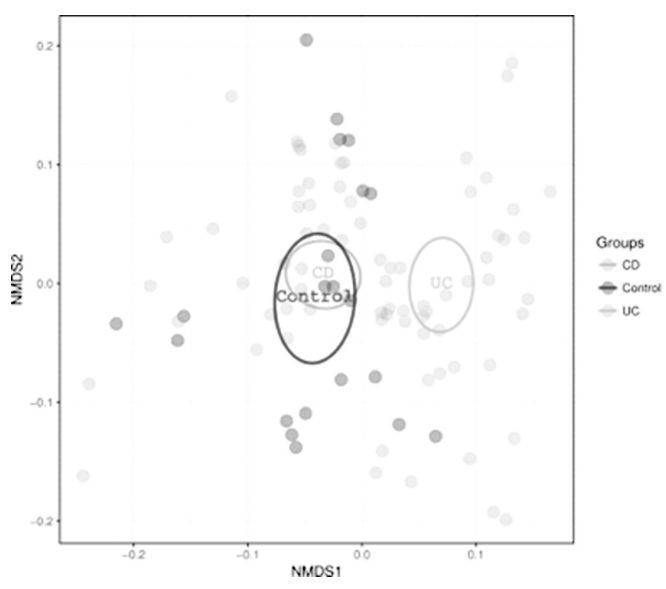

Abstract OWE-010 Figure 5 NMDS weighted UniFrac (bacteria-all biopsies - diagnosis) $R^{2}=0.11 p=0.001$

The mucosal bacterial community was dysbiotic and influenced by subject, disease distribution (figure 4) and diagnosis (figure 5). In terms of mycobiome, fewer viable reads were obtained, due to limited template. Saccharomyces was the most abundant fungi, but it was absent in some samples, other relevant genera were Malassezia, Cladosporium, Aspergillus and Candida. The last was found more often in controls.

Conclusions CD patients' bacterial community is dysbiotic but fungi are not. Saccharomyces dominated most of faecal samples, but not biopsies. This implies it may not be a permanent resident of the mucosae. Fungi may arise from food: it is hard to discriminate what comes from food and what is active in the gut. The concept of a resident, symbiotic mycobiome needs further exploration.

\section{OWE-011 CLINICAL EFFECTIVENESS, SAFETY AND IMMUNOGENICITY OF ANTI-TNF THERAPY IN CROHN'S DISEASE: 12-MONTH DATA FROM PANTS}

${ }^{1}$ Nicholas Kennedy*, ${ }^{1,2}$ Graham Heap, ${ }^{1}$ Gareth Walker, ${ }^{1}$ Claire Bewshea, ${ }^{2}$ Sonia Bouri, ${ }^{1}$ James Goodhand, PANTS investigator consortium, 'Tariq Ahmad. 'IBD Pharmacogenetics, University of Exeter, Exeter, UK; ${ }^{2}$ Abbvie, Chicago, USA; ${ }^{3}$ BBD Unit, St Mark's Hospital, London, UK

10.1136/gutjnl-2018-BSGAbstracts.116
Introduction PANTS (Personalised Anti-TNF Therapy in Crohn's disease [CD]) is a 3 year prospective observational UK-wide study investigating primary non-response (PNR), loss of response and adverse drug reactions to infliximab (IFX: Remicade [REM], CT-P13) and adalimumab (ADL: Humira). We now report the wk 54 clinical effectiveness and safety outcomes, and immunogenicity data to date.

Methods Inclusion criteria included: CD patients aged $\geq 6$ years, active inflammatory disease (raised CRP [>3 mg/L] or calprotectin $[\geq 50 \mu \mathrm{g} / \mathrm{g}])$ and no prior anti-TNF therapy. PNR was defined at wk $12-14$ as a requirement for ongoing steroids, or both HBI failed to fall by $\geq 3$ points or to $\leq 4$ and CRP failed to fall by $\geq 50 \%$ or to $\leq 3 \mathrm{mg} / \mathrm{L}$. Remission was defined at wks 14 and 54 as $\mathrm{HBI} \leq 3$ points and CRP $\leq 3 \mathrm{mg} / \mathrm{L}$ and no concomitant steroids. Patients who stopped drug other than for elective withdrawal, pregnancy or loss to follow-up were regarded as treatment failures for subsequent endpoints. Drug (DL) and anti-drug antibody (ADA) levels were measured using the IDKmonitor drug tolerant assays. Immunogenicity was defined as ADA titre $\geq 10 \mathrm{AU} / \mathrm{ml}$ + undetectable DL.

Results 1601 (49\% male, median age 33 years [IQR 23-47]) eligible patients were recruited from 118 sites. Patients were treated with IFX (751 [47\%]: REM, 200 [12\%] CT-P13) or ADL (650 [41\%]). Baseline characteristics included: median disease duration 3 years (IQR 1-10); steroids 27\%, azathioprine 44\%, mercaptopurine $8 \%$, methotrexate 5\%; median $\mathrm{CRP}$ in IFX $9 \mathrm{mg} / \mathrm{L}$ (IQR CI 3-24) and $6 \mathrm{mg} / \mathrm{L}$ (IQR 2-14) in ADL. PNR at week 12-14 was $21 \%, 21 \%$ and $26 \%$ in the REM, CT-P13 and ADL treated patients respectively. PNR was associated with older age $(p=0.0004)$, higher BMI $(p=0.03)$ and low DL $(\mathrm{p}<0.0001$ for IFX and ADL). Week 54 remission rate was $40 \%, 40 \%$ and $34 \%$ of the REM, CT-P13 and ADL treated patients. At wk 54, the immunogenicity rate for REM, CT-P13 and ADL was 26\%, 28\% and $11 \%$ rising to $42 \%, 38 \%$ and $23 \%$ by 3 years respectively (IFX vs. ADL $\mathrm{p}<0.0001$, REM vs. CT-P13 $\mathrm{p}=0.25)$. Immunogenicity was associated with non-remission at wk $54(\mathrm{p}<0.0001$ for both IFX and $\mathrm{ADL})$. Immunomodulator use reduced the risk of immunogenicity for both IFX $(\mathrm{HR}=0.37, \mathrm{p}<0.0001)$ and ADL $(\mathrm{HR}=0.34, \mathrm{p}<0.0001) .140$ patients $(9 \%)$ withdrew drug for SAEs including 5 who died, 3 from CD and 2 from possibly drug-related acute respiratory illness.

Conclusions This is the largest prospective real-life study of anti-TNF therapy in IBD. We report the clinical effectiveness, safety and immunogenicity of REM, CT-P13, and ADL. This cohort provides a unique bioresource for multi-omic studies investigating personalised approaches to anti-TNF therapy.

\section{OTH-003 PAEDIATRIC CROHN'S DISEASE PATIENTS IN REMISSION HAVE A REDUCED SKELETAL MUSCLE PROTEIN BALANCE AFTER FEEDING}

${ }^{1,2}$ Gordon Moran, ${ }^{3}$ Amanda Walker, ${ }^{3}$ Aline Nixon, ${ }^{1}$ David Devadason, ${ }^{4}$ Rafeeq Muhammed,
${ }^{3}$ Kostas Tsintzas, ${ }^{1}$ Sian Kirkham, ${ }^{5}$ Francis Stephens. ${ }^{1}$ Nottingham University Hospitals NHS
Trust; ${ }^{2}$ NIHR Nottingham Biomedical Research Centre; ${ }^{3}$ School of Life Sciences, University of
Nottingham; ${ }^{4}$ Birmingham Children's Hospitals; ${ }^{5}$ Sports and Health Sciences, University of
Exeter

10.1136/gutjnl-2018-BSGAbstracts. 117

Introduction Sarcopenia is common in active Crohn's disease (CD) and still prevalent in remission. This can lead to fatigue, physical inactivity and poor quality of life but the aetiology is 
unclear. We aimed to investigate the association between sarcopenia and anabolic resistance (AR) and insulin resistance (IR), and the role of physical activity in age, gender matched children with CD.

Methods 18 fasted, male and female CD (on thiopurines \pm anti$\mathrm{TNF} \alpha)$ in deep remission (16 y, BMI=21) and 9 matched controls (Con) (16 y, BMI=21) drank a liquid meal (Ensure plus, $44 \mathrm{~g} \mathrm{CHO}, 14 \mathrm{~g}$ PRO, $11 \mathrm{~g}$ fat) at $\mathrm{t}=0$. Arterialised hand and venous forearm blood samples were collected concurrently and brachial artery blood flow measured at baseline and every 20 mins for 2 hours. Net balance of branched chain amino acids (BCAA) and glucose were derived, giving indices of skeletal muscle protein balance and IR. Subjects had a DEXA scan and handgrip dynamometer test on the day, and wore a pedometer and completed a food diary (for 3 days) to assess physical activity and food intake. Patient questionnaires (incl. IBD-fatigue) were completed.

Results Net BCAA balance across the whole 2 hours was lower in $C D$ vs Con $(-0.1 \pm 0.2 \mu \mathrm{mol} / \mathrm{min}$ vs $0.6 \pm 0.3 \mu \mathrm{mol} /$ $\min , p=0.05)$. Yet an initial response to feeding $(t=0$ to $\mathrm{t}=20)$ was exhibited by both $\mathrm{CD}(+1 \mu \mathrm{mol} / \mathrm{min})$ and Con $(+0.8 \mu \mathrm{mol} / \mathrm{min})$ but was only sustained post $40 \mathrm{mins}$ in Con. IBD-fatigue scores indicated CD had moderate fatigue (6), which had a moderate effect on daily activities (17). Handgrip dynamometer testing showed a trend towards greater fatigue in $\mathrm{CD}$ vs Con (+8\%pts) in the dominant arm $(\mathrm{p}=0.061)$. A trend towards lower total body lean mass in CD $(-15 \%$, $\mathrm{p}=0.084$ ) was found. No differences were detected in strength, physical activity, diet or IR. Thus despite not exhibiting AR (initial response to the meal) CD could not maintain a positive protein balance post feeding. This was associated with reduced muscle mass and function.

Conclusions The inability to sustain a positive protein balance postprandially could provide an explanation for the reduced muscle mass seen in $\mathrm{CD}$ patients in remission and be contributing to fatigue and poor muscle function. Pharmacological interventions to reduce protein breakdown and a high protein diet and /or exercise to improve anabolic response could be investigated as potential treatments.

\section{ADTU-02 V565, A NOVEL ORAL ANTI-TNF DOMAIN ANTIBODY, REDUCES COLONIC MUCOSAL INFLAMMATION IN PATIENTS WITH UC}

${ }^{1}$ Gareth Parkes*, ${ }^{2}$ Suhail Nurbhai, ${ }^{2}$ Mike West, ${ }^{2} \mathrm{~K}$ Ray, ${ }^{3} \mathrm{Tom}$ MacDonald ${ }^{3}$ Anna Vossenkaemper, ${ }^{2} S$ Crowe. ${ }^{1}$ Royal London Hospital, London, UK; ${ }^{2}$ VHSquared, Cambridge, UK; ${ }^{3}$ Centre for Immunobiology, Blizard Institute, QMUL, London, UK

\subsection{6/gutjnl-2018-BSGAbstracts.118}

Introduction Monoclonal antibodies to TNF transformed treatment options for patients with Inflammatory Bowel Disease (IBD). V565 is a novel oral anti-TNF domain antibody (Vorabody) engineered to be resistant to intestinal proteases. It is in development as a potential oral treatment for IBD. In vitro it suppressed phosphorylation of tyrosine kinases and signalling proteins and inhibited the release of inflammatory cytokines following culture with biopsies taken from patients with CD (Crowe et al. 18th International Congress of Mucosal Immunology, July 19-22 2017, Washington DC, USA). It was safe and well tolerated after high single and multiple doses in healthy volunteers and patient volunteers with $\mathrm{CD}$ and resulted in high concentrations of active drug in ileal fluid and faeces.
Aims \& Methods This open label study was designed to demonstrate that V565 enters GI mucosa and exerts a beneficial effect on inflammatory processes following oral dosing for 7 days to patients with Ulcerative Colitis. Patients with a Mayo score of 3-10 including an endoscopy score of $\geq 1$ had up to 7 days of oral dosing with $555 \mathrm{mg}$ tid V565. Sigmoidoscopy with biopsies was performed before and after the dosing period. The primary outcomes of interest were presence of V565 in the mucosa and reduction from baseline in phosphorylation of tyrosine kinases and signalling proteins. Detection of V565 was determined by immunohistochemistry. Phosphorylation was determined using PathScan RTK signalling arrays (Vossenkaemper et al 2014. Gastroenterology 147:172-83).

Results

Five patient volunteers were treated Due to visit scheduling, most received 6 days treatment. Presence of V565 was confirmed in the inflamed lamina propria and co-localised with CD14 + macrophages in post-treatment biopsies. Overall phosphorylation of the panel of kinases and signalling proteins was reduced by approximately $50 \%$ in four of the five patients. There were no treatment induced ADAs.

Conclusion V565, an oral anti-TNF domain antibody engineered to be resistant to intestinal proteases, was demonstrated bound to CD14 +macrophages in the lamina propria of UC patients and resulted in inhibition of mucosal inflammatory processes after 6-7 days oral dosing. The reduction of $50 \%$ in overall phosphorylation is similar to that seen in an earlier study of UC biopsy cultures with infliximab at a concentration of $67 \mathrm{nM}(10 \mu \mathrm{g} / \mathrm{ml})$, a serum concentration associated with mucosal healing (Ungar et al, Clin Gastroenterol Hepatol. 2016 Apr;14(4):550-557). These results provide encouragement that oral dosing with V565 will be a beneficial oral treatment option for patients with IBD.

\section{ADTU-03 IMPACT OF IMPROVED ACCESS TO BIOLOGIC THERAPIES AND PHYSICIAN ENGAGEMENT ON EXCESS STEROID EXPOSURE}

Gareth Parkes*, Christian Selinger, Ash Bassi, Melissa Smith, Jimmi Lindi, Helen Ludlow, Pritash Patel, Santosh Salunke, Veronica Hall, Becky George, John Saunders, Mark Adamson, Fiona Donovan, loanna Parisi, Lynn Gray, Aileen Fraser, Richard Pollock, Glyn Scott, Tim Raine. IBD Steroid Assessment Tool Group, Various, UK

\subsection{6/gutjnl-2018-BSGAbstracts. 119}

Background Steroid free remission is an important goal of IBD therapy. The aim of this study was to evaluate temporal changes in steroid prescribing in UK IBD outpatients in the context of major changes in UK prescribing guidelines and physician participation in audit and tailored service changes.

Methods Steroid use over the previous 12 months was recorded for unselected outpatient attenders against a definition of excess from ECCO guidelines. Data were collected from 7 centres that had completed a steroid assessment audit cycle in 2015, as well as from 12 new matched centres.

Results Data was collected for 2385 patients May-July 2017 and compared with 2015 data from 1176 patients. Overall disease distribution was $47.1 \% \mathrm{CD}, 49.6 \% \mathrm{UC}$ and $3.3 \%$ IBD-U, whilst $77.7 \%$ of patients were in clinical remission at the time of assessment. There was only a modest increase in patient exposure to anti-TNF from 2015 to 2017 : $30.6 \%$ to $37.2 \%$ in CD $(p=0.009)$ and $9.9 \%$ to $12.0 \%$ in UC $(p=N S)$. Anti-integrin usage increased from $0.8 \%$ to $3.3 \%$ in $\mathrm{CD}$ $(p=0.002)$ and from $1.6 \%$ to $2.4 \%$ in UC $(p=N S)$. For 